\title{
Goal Orientated Stroke Rehabilitation Utilising Electrical Stimulation, Iterative Learning and Microsoft Kinect
}

\author{
Timothy Exell, Christopher Freeman, Katie \\ Meadmore, Mustafa Kutlu and Eric Rogers \\ Electronics and Computer Science \\ University of Southampton \\ Southampton, UK \\ SO17 1BJ \\ Email: te1@ecs.soton.ac.uk
}

\author{
Ann-Marie Hughes, Emma Hallewell \\ and Jane Burridge \\ Faculty of Health Science \\ University of Southampton \\ Southampton, UK \\ SO17 1BJ
}

\begin{abstract}
An upper-limb stroke rehabilitation system is developed that assists patients in performing real world functionally relevant reaching tasks. The system provides de-weighting of the arm via a simple spring support whilst functional electrical stimulation is applied to the anterior deltoid and triceps via surface electrodes, and to the wrist and hand extensors via a 40 element surface electrode array. Iterative learning control (ILC) is used to mediate the electrical stimulation, and updates the stimulation signal applied to each muscle group based on the error between the ideal and actual movement in the previous attempt. The control system applies the minimum amount of stimulation required, maximising voluntary effort. Low-cost, markerless motion tracking is provided via a Microsoft Kinect, with hand and wrist data provided by an electrogoniometer or data glove. The system is described and initial experimental results are presented for a stroke patient starting treatment.
\end{abstract}

Keywords: Robotics; Electrical stimulation; Iterative learning control.

\section{INTRODUCTION}

Stroke is the largest cause of adult disability in the UK, affecting 110,000 people every year [1]. Less than $15 \%$ of patients that experience initial upper-limb paralysis following stroke regain full function [2], which restricts their ability to perform everyday reaching and grasping tasks.

Functional electrical stimulation (FES) can assist stroke patients in moving their impaired limbs, and its use in rehabilitation has been shown to increase upper-limb function [3]. Benefits of FES have been shown to be greatest when combined with maximal voluntary effort from the patient to perform the movement [4]. Rushton [5] hypothesized that the application of FES to assist movements that the patient is attempting to perform with voluntary effort may lead to Hebb-type relearning and greater long-term recovery. It is hence important for rehabilitation systems to provide the minimum amount of FES required to accurately assist the movement, encouraging the patient's maximum voluntary effort.

To achieve precise movement, a technique called iterative learning control (ILC) has been used to adjust the FES.
This is a control method originally developed for industrial robots repeatedly performing a pre-defined movement. The data collected throughout each repetition of the movement is then used to update the control input signal for the next execution with the objective of increasing the accuracy of the movement. Recent work has successfully used ILC to regulate the timing and intensity of FES applied to stroke patient's muscles whilst performing planar [6], [7] and 3D [8], [9], [10] reaching tasks. These previous studies reported significant improvements in both performance accuracy and clinical outcome measures of movement for stroke patients following clinical trials, demonstrating the potential of ILC and FES during rehabilitation. The system developed in this paper progresses the application of ILC and FES to realworld tasks incorporating the hand and wrist, and reduces the cost and equipment demands of the system, partly through use of the Microsoft Kinect ${ }^{\circledR}$. It thereby advances towards a rehabilitation system that may be used in patients' homes.

\section{System OVERVIEW}

Previous studies combining ILC and FES for upper limb rehabilitation have used planar light tracking tasks [6] or virtual reality $3 \mathrm{D}$ object tracking [8], incorporating explicit reference trajectories for the patient to follow. To directly target activities of daily living, the current system assists real-world tasks that require manipulation of objects using the hand and arm. The optional addition of an electrode array for finer control of hand and wrist motion is also included. Support against gravity is provided for the patient's arm using a commercially available passive spring support (SaeboMAS®, Saebo, Charlotte, USA). Tracking of the patient's arm and hand movements is achieved using a Kinect ${ }^{\circledR}$ (Microsoft, Washington, USA) motion capture device and wrist electrogoniometer (Biometrics Ltd, Newport, UK). An optional 5DT data glove (5DT Inc, California, USA) is also used for collection of finger movement, where required. Since no explicit reference trajectory is shown to 
the patient, the ILC scheme employs principles from motor control to deliver the optimum FES assistance [11].

\section{A. Motion Tracking}

The recent release of the Kinect has revolutionised noninvasive motion capture by providing a free software development kit and pre-calibrated 'out of the box' hardware, which has vastly reduced the associated hardware and software cost [12]. The Kinect is a small $(0.30 \mathrm{x} 0.08 \mathrm{x}$ $0.06 \mathrm{~m})$, lightweight $(1.4 \mathrm{~kg})$ device incorporating a video camera with an infra-red source and an infra-red sensor. The infra-red sensor measures the reflection of infra-red light by objects in front of the camera and calculates 3D position data of those objects. Recent accuracy tests have indicated that the device is capable of calculating position data with an accuracy of around $1 \mathrm{~cm}$ [13]. The software supplied with the Kinect uses pattern recognition to detect landmarks of interest, such as limb segments and joint estimations.

In this system, the Kinect is used to capture joint centre locations for the shoulder, elbow and wrist, for the calculation of shoulder and elbow joint angles. It is not possible to use the Kinect for the calculation of wrist and hand joint angles, due to accuracy limitations in hand tracking when the device is at the distance required to produce a large enough field of view for all the other segments. Therefore, an electrogoniometer is included to collect wrist angle data and an optional data glove can be used to capture individual finger joint angle data.

\section{B. FES Module}

FES surface electrodes are positioned on the patient's anterior deltoid, triceps and wrist and hand extensor muscles, with placement following clinical guidelines. A series of $5 \mathrm{~V}, 40 \mathrm{~Hz}$ pulses are produced by the control hardware for each channel and amplified by a four-channel electrical stimulator (Odstock Medical Limited, Salisbury, UK) to generate a bi-phasic signal which achieves a smooth muscle contraction [14]. For safety, the maximum pulsewidth that can be applied to any channel is limited within the control software and also by the stimulator. Prior to each session, the amplification level for each channel is set by applying a constant stimulation signal with pulsewidth $300 \mu$ s from the control hardware and slowly increasing the voltage until the maximum comfortable level is reached. During subsequent tests the pulsewidth is limited to $300 \mu \mathrm{s}$.

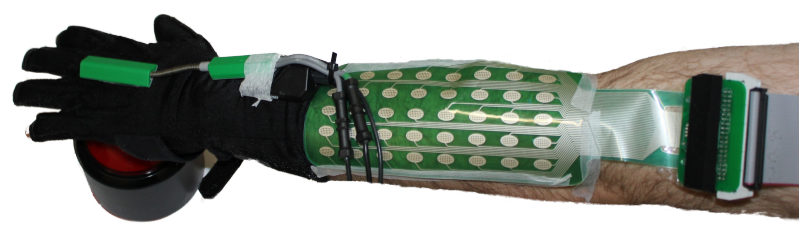

Fig. 1. Electrode array with data glove and electrogoniometer used for stimulating and tracking hand and wrist movement.

\section{Electrode Array}

The electrode array (Fatronik, Spain) comprises 5 x 8 elements which can each be routed to one of four FES channels. This is achieved by custom made RS232 controlled multiplexor hardware, comprising an Arduino board and shift register array. For each required hand and wrist posture, the optimal electrode sites and associated FES pulsewidths are selected during initial tests through a search procedure involving local linear model identification and gradientdescent optimization [15]. The sites are then fixed during subsequent experiments, with the pulsewidth amplitudes then controlled by ILC. Note, due to the additional difficulty in using the data glove, the electrode array can be replaced by a pair of standard electrode pads depending on patient need. The electrode array and data glove are shown in Figure 1.

\section{System Software}

The system software incorporates tracking of the patient's movement with the control schemes implemented to mediate the FES in real-time. A custom made $\mathrm{C}++$ application is used to read the arm and hand positions from the Kinect, electrogoniometer and data glove. Position data are then transferred to the real-time control hardware (dSPACE ds1103) which implements ILC schemes involving embedded dynamic models of the arm. Outputs of the control scheme comprise pulse-width modulated (PWM) signals for each of the FES stimulator channels, together with RS232 serial data to control the electrode array. Digital inputs and outputs are also employed to interface with the instrumented task objects. A signal flow diagram is shown in Figure 2.

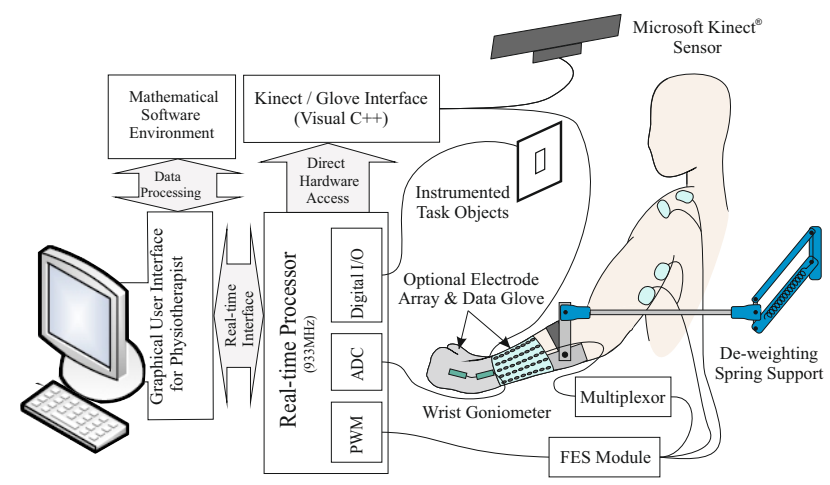

Fig. 2. Signal flow diagram.

A graphical user interface (GUI) has been developed to oversee the system inputs and outputs and is responsible for customising control parameters, implementing the FES control, collecting position outcome data, selecting the task details to be performed and reviewing performance after each session. During treatment, each patient attends $18 \mathrm{x}$ 1 hour sessions during which the system assists them in performing functional reaching and grasping tasks. Prior to each session, a physiotherapist assists the patient into the de-weighting support and places surface electrodes at the required positions of the patient's extensor muscles of the shoulder, elbow, wrist and hand. The physiotherapist 
uses a hand-held electrical stimulator when positioning the electrodes so that they are placed at the optimal locations to produce the desired movement. When incorporating the electrode array, the optimum stimulation location for the wrist and hand is automated by the software (see Section II-C). The maximal FES levels are set for all electrode channels so that they provide the required amount of stimulation to achieve the required movement, whilst not causing discomfort to the patient (see Section II-B). Tests are then undertaken to identify parameters in the dynamic model of the patient's arm (see Section III).

At the beginning and end of each treatment session, four 'button pushing' tasks are performed without any FES being applied (see Section II-E). The patient's performance during the unassisted 'button pushing' tasks is used to monitor their arm function over time. Following the unassisted tasks, the physiotherapist selects the tasks to be performed with stimulation, based on the specific rehabilitation requirements of each patient. Each task is typically performed 6 times with 20 s rest time between each trial. Immediately following each trial and before the next attempt, the magnitude and timing of the FES applied to each muscle group is updated by the ILC scheme, based on the error contained during the preceding attempt.

Sensors contained within the task objects are used to detect if the patient has completed the task, at which point the FES is terminated for all muscle groups so that the patient can return their hand to the starting position. The physiotherapist also has a safety button that they can press to immediately stop the stimulation during a trial.

\section{E. Reaching Tasks}

Evolving from the previous systems that used planar light tracking [6] or virtual reality 3D object tracking tasks [8], this system uses functionally relevant real world tasks. The tasks include: (1) switching a low light switch (shoulder height), (2) switching a high light switch (head height), (3) closing a drawer (shoulder height), (4) stabilising an object on a table with the affected arm whilst manipulating the object with the unaffected arm, (5) repositioning a drinksized object on a table, (6) pressing buttons positioned on a table. All of the task endpoints are scaled for each patient's arm length and shoulder height so that the reference joint angle signals result in the desired positioning of the hand for the task. Figure 3 shows the workstation set up for the functional tasks; the tasks were selected so that they incorporate different aspects of reaching, grasping and manipulating objects at varying elevation ranging from table height to head height.

\section{ARM MODEL AND CONTROL}

A dynamic model of the arm-support system incorporates a biomechanical description of the human arm and a representation of the SaeboMAS spring support. Figure 4 shows a block diagram of the control system and arm model.
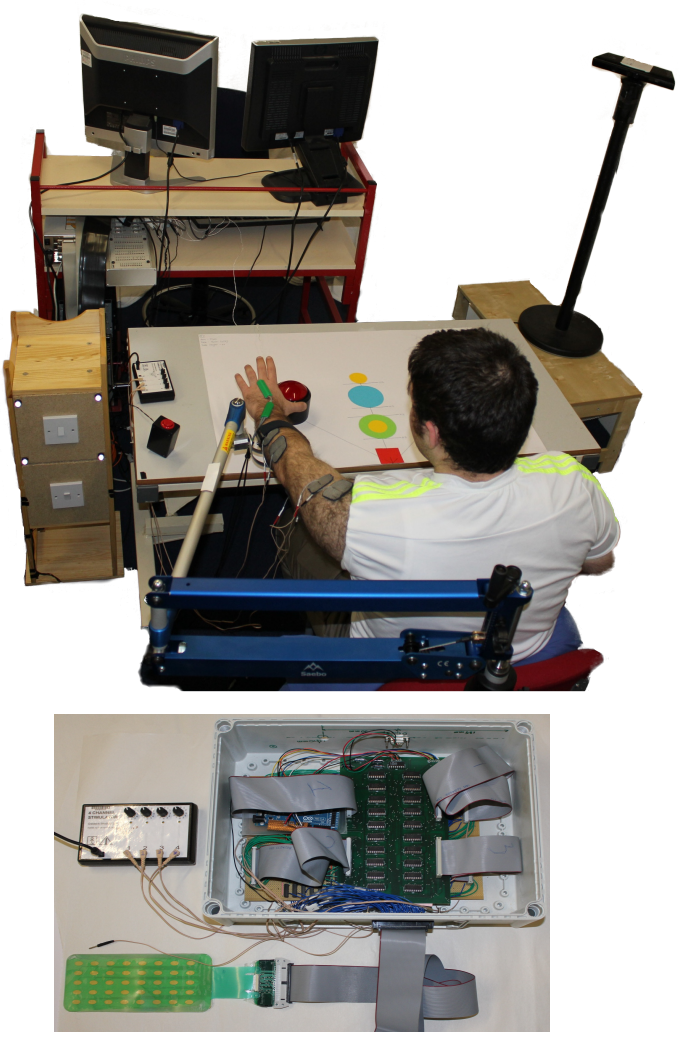

Fig. 3. Functional task workstation (top) and optional electrode array and hardware (bottom).

\section{A. Human Arm Model}

Position values for the shoulder, elbow and wrist joint centres are calculated using the Kinect. To assist the FES control scheme, a simplified model of the arm is used for the calculation of joint angles. Figure 5 shows the kinematic model of the human arm. Spasticity in stroke patients often restricts flexion of the shoulder in the anteroposterior plane, extension of the elbow and extension of the wrist and fingers [16]. Therefore, the anterior deltoid, triceps and wrist and hand extensor muscles were selected for stimulation. It is assumed that stimulation applied to the triceps produces movement about an axis perpendicular to the upper and forearm segments and that stimulation applied to the wrist and hand extensors produces movement about an axes that is fixed with respect to the forearm. For the anterior deltoid it is assumed that stimulation produces movement about an axis that is fixed with respect to the shoulder and determined by two rotation transformations. These comprise rotations around the $z$-axis by $\beta$ and around the $x$-axis by $\gamma$. Identification of $\beta$ and $\gamma$ is described in Section III-C.

The dynamic model of the human arm is given in the simplest case (excluding a full model of the hand) by

$$
\begin{array}{r}
B_{h}(\Phi) \ddot{\Phi}+C_{h}(\Phi, \dot{\Phi}) \dot{\Phi}+F_{h}(\Phi, \dot{\Phi})+G_{h}(\Phi) \\
=g(u, \Phi, \dot{\Phi})-J^{T}(\Phi) h_{h}
\end{array}
$$

where $B_{h}(\cdot)$ and $C_{h}(\cdot)$ are 6-by-6 inertial and Coriolis matrices respectively, $F_{h}(\cdot)$ and $G_{h}(\cdot)$ 


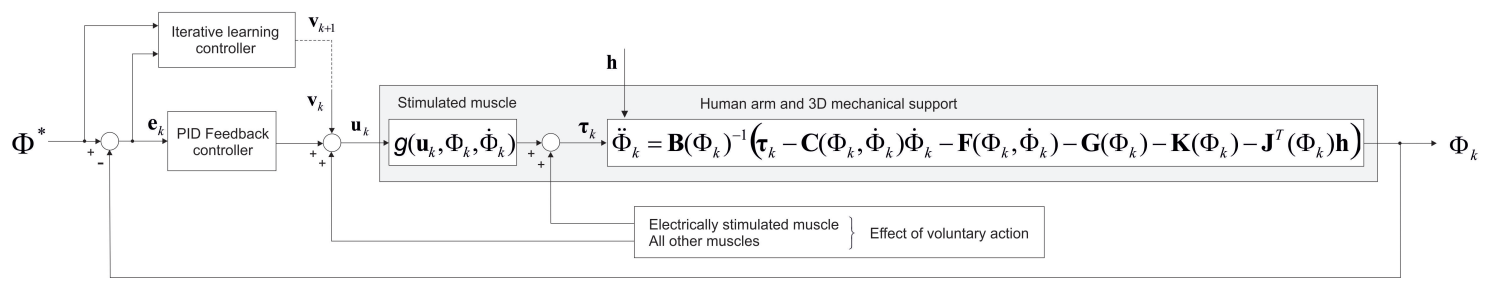

Fig. 4. Block diagram of the ILC scheme.

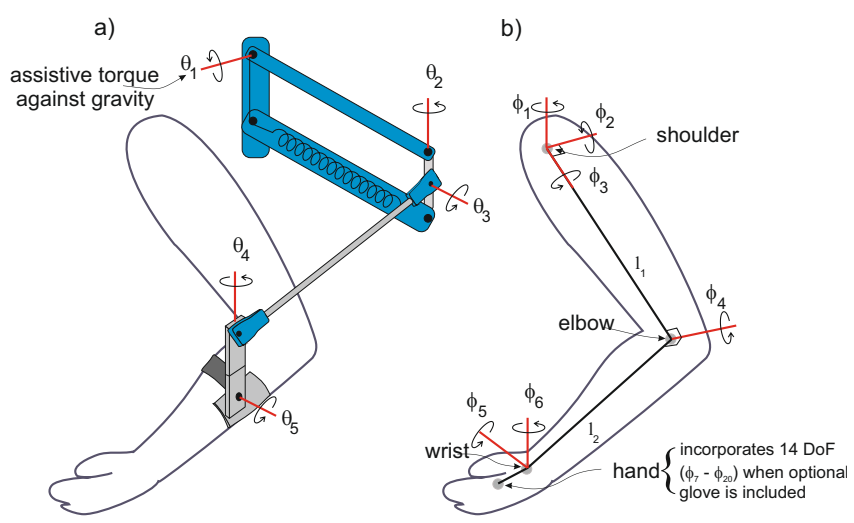

Fig. 5. Kinematic model of the (a) SaeboMAS support and (b) human arm.

are friction and gravitational vectors and $g(\cdot)$ comprises the moments produced through application of FES, which are of the form $g(u, \Phi, \dot{\Phi})=$ $\left[g_{1}\left(\phi_{1}, \dot{\phi}_{1}, u_{1}\right), 0,0, g_{4}\left(\phi_{4}, \dot{\phi}_{4}, u_{4}\right), g_{5}\left(\phi_{5}, \dot{\phi}_{5}, u_{5}\right), 0\right]^{T}$.

Here $h_{h}$ is a vector of externally applied force and torque comprising components $h_{s}$ due to the spring support and $h$ due to interaction with objects; $J(\cdot)$ is the system Jacobian. Moreover $u_{1}(t), u_{4}(t)$ and $u_{5}(t)$ represent the electrical stimulation applied to the anterior deltoid, triceps and wrist and hand extensor muscles, respectively with $u=\left[u_{1}, 0,0, u_{4}, u_{5}, 0\right]^{T}$. From [17], each moment can be assumed to be of the form

$g_{i}\left(\phi_{i}, \dot{\phi}_{i}, u_{i}(t)\right)=h_{i}\left(u_{i}, t\right) \times F_{m, i}\left(\phi_{i}, \dot{\phi}_{i}\right) \quad i \in\{1,4,5\}$

The term, $h_{i}\left(u_{i}, t\right)$ is a Hammerstein structure incorporating a static non-linearity, $h_{I R C, i}\left(u_{i}\right)$, that represents the isometric recruitment curve, cascaded with linear activation dynamics, $h_{L A D, i}(t)$. The term $F_{m, i}\left(\phi_{i}, \dot{\phi}_{i}\right)$ models the multiplicative effect of the joint angle and joint angular velocity on the active torque developed by the muscle.

The SaeboMAS support structure has the form

$$
\begin{array}{r}
B_{s}(\Theta) \ddot{\Theta}+C_{s}(\Theta, \dot{\Theta}) \dot{\Theta}+F_{s}(\Theta, \dot{\Theta})+G_{s}(\Theta)+K_{s}(\Theta) \\
=-J_{s}^{T}(\Theta) h_{s}
\end{array}
$$

where $B_{s}(\cdot)$ and $C_{s}(\cdot)$ are 5-by-5 inertial and Corelis matrices, $\Theta=\theta_{1} \ldots \theta_{5}$, representing the angles of the spring support. In addition, $J_{s}(\cdot)$ is the system Jacobian, and $F_{s}(\cdot)$ and $G_{s}(\cdot)$ are friction and gravitational vectors. The vector $K_{s}(\cdot)$ comprises the moments produced through gravity compensation provided by the spring, which takes the form $\left[k_{1}\left(\theta_{1}\right), 0,0,0,0\right]^{T}$. The rigid connection between structures gives rise to bijective mapping between $\Phi$ and $\Theta$ so that the combined model is given by

$$
\begin{array}{r}
B(\Phi) \ddot{\Phi}+C(\Phi, \dot{\Phi}) \dot{\Phi}+F(\Phi, \dot{\Phi})+G(\Phi)+K(\Phi) \\
=g(u, \Phi, \dot{\Phi})-J^{T}(\Phi) h
\end{array}
$$

This model of the arm is used by the FES control system to produce an input signal that results in accurate completion of the tasks. During trials incorporating FES, the controller assists tracking about $\phi_{1}, \phi_{4}$ and $\phi_{5}$ alone, and it is assumed that the patient has sufficient control over the remaining axes to adequately perform the task.

\section{B. Hand Model}

Due to the complexity of identifying parameters in a full dynamic model of the hand and wrist, the array element identification procedure uses stimulation and angular output data from the glove to construct a linear model linking these variables. These are then integrated with the simpler model described in (1). See [15] for full details.

\section{Model Identification}

The FES control schemes utilise a dynamic model of the combined human arm and mechanical support, so that stimulation results in accurate tracking of the reference profiles calculated for each task. The dynamic model requires the two parameters, $\beta$ and $\gamma$, which define the anterior deltoid axis. These parameters are determined by applying a ramped $10 \mathrm{~s}$ FES signal to the anterior deltoid and recording the associated movement of the patient's elbow. It is assumed that the spring support cancels the effect of gravity so that the stimulation only produces movement about the anterior deltoid axis. A plane is fitted to the elbow positions that were collected whilst the stimulation was applied, which is then used to determine $\beta$ and $\gamma$.

\section{FES Control Strategy}

An advanced control system utilising constrained pointto-point optimisation has been developed and experimentally verified [11]. This novel approach to controlling movement embeds results from human motor control and poses each task as a constrained optimisation problem, such as moving from one point to another with constraints applied on the joint velocity and acceleration to ensure a smooth movement. In the ILC framework these problems are solved iteratively using joint data from each attempt to update the control input [18], [19], [20]. This improves on using predefined 
references for each task since (1) the task is tailored to each patient using their underlying arm dynamics, and (2) new tasks can be added without requiring predefined references to have been collected. A limitation of implementing this technique is the requirement of a full dynamic model, which entails a lengthy experimental procedure. Therefore, to minimise cost and maximise usability of the system, a simpler approach termed phase-lead ILC is currently used in this system, based on tracking joint references extracted from predetermined movement data.

The control system shown in Figure 4 is used to assist tracking of reference signals that are extracted for the shoulder, elbow and wrist joints. Control is implemented for the pulse width inputs $u_{1}(t), u_{4}(t)$ and $u_{5}(t)$ in the input vector $u(t)$. The outputs controlled by the system are the elements $\phi_{1}(t), \phi_{4}(t)$ and $\phi_{5}(t)$ of vector $\Phi(t)$, which track the corresponding elements $\phi_{1}^{*}(t), \phi_{4}^{*}(t)$ and $\phi_{5}^{*}(t)$ of $\Phi^{*}(t)$ containing the joint angle reference signals for each task. Joint angles not controlled by the system can either be assumed fixed and removed, or treated as a disturbance.

The reach and grasp tasks that are incorporated in the system consist of repeated movements for the patient's affected arm, with a rest period in between during which their arm is returned to a common starting position. The repetitive performance of finite duration tasks used in this system allow ILC to be utilised to control the FES signals for each muscle group. Each ILC trial starts from a fixed initial hand position and the performance error from each trial is used to update the control parameters in an attempt to increase accuracy of the subsequent performance. In the current system, a proportional-integral-derivative (PID) controller is typically utilised in parallel with the ILC update, shown in Figure 4. Using this control structure, voluntary movement by the patient can be treated as iteration-invariant disturbance and can be compensated for [21]. A robust ILC scheme can deal with dynamic changes and model inaccuracy due to fatigue, spasticity and other physiological effects [6]. The phase-lead ILC algorithm has the form

$$
v_{k+1}(t)=v_{k}(t)+L e_{k}(t+\tau)
$$

where $L$ is a scalar learning gain, $\tau$ is the phase-lead parameter and $v_{k+1}$ is added to the feedback control output to produce stimulation update $u_{k+1}$. The joint error $e_{k}$ is calculated from

$$
e_{k}(t)=\Phi^{*}(t)-\Phi_{k}(t)
$$

where $\Phi^{*}$ is the reference trajectory. Since only three joint angles $\left(\phi_{1}, \phi_{4}\right.$ and $\left.\phi_{5}\right)$ are controlled, $L$ is multiplied by the matrix $\{1,0,0,1,1,0\}$. The term $\tau$ is typically chosen as the time to maximum impulse response and can be determined experimentally using a step or ramp response [22].

Since (2) requires a reference signal, unimpaired trials have been collected for the functional tasks. Reference signals were collected for the three controlled joint angles and tasks were scaled to the size and reach of each patient. Variation in the joint angles $\phi_{2}$ and $\phi_{3}$ is allowed by the
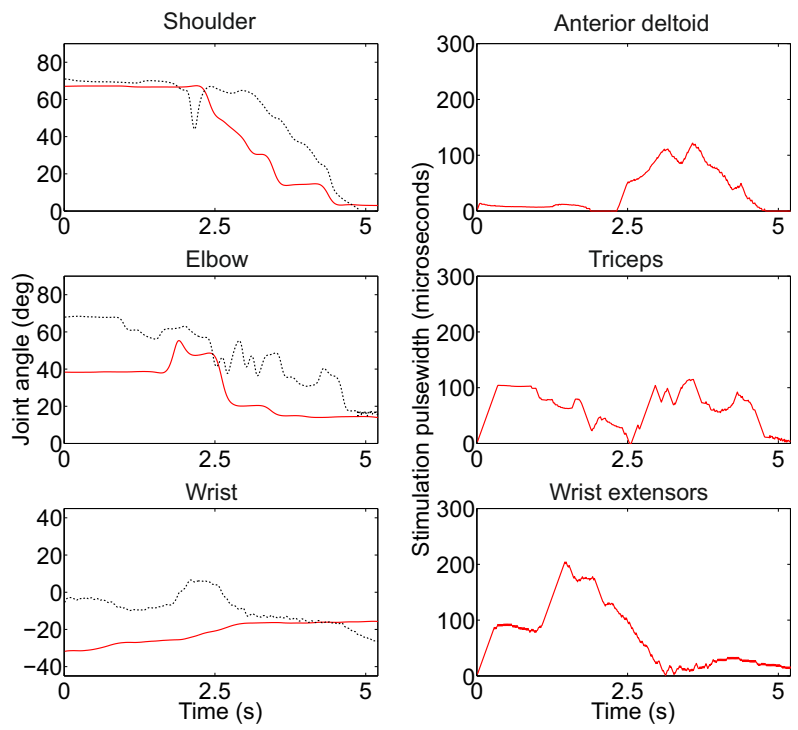

Fig. 6. Example data from far button task. Left: Patient performance for shoulder, elbow and wrist joint (reference angles = solid, patient performance $=$ dashed); Right: Stimulation applied to each muscle group.

system since only $\phi_{1}, \phi_{4}$ and $\phi_{5}$ are controlled. Tasks were included in the system that are functionally relevant to daily reach and grasp tasks and offer a range of movement difficulties in 3D space. The tasks that are comprise each rehabilitation session are selected by the physiotherapist, based on the individual requirements of the patient.

\section{EXPERIMENTAL RESULTS}

Ethical approval was obtained from the University's ethics committee. Clinical trials are currently underway and initial results are available from the first three patients. Data were collected at $100 \mathrm{~Hz}$ for the wrist electrogoniometer and data glove, position data collected using the Kinect were limited to a lower sampling frequency (typically $33 \mathrm{~Hz}$ ), and interpolated to $100 \mathrm{~Hz}$. Control signals for the FES were sampled at $40 \mathrm{~Hz}$, as required for the FES.

The patients performed the 'button pushing' and 'high light switch' tasks without any FES at the start of each session. Following the unassisted tests, patients performed a selection of the tasks with FES assistance, mediated by the ILC algorithms. Maximum voluntary effort was encouraged during the FES assisted trials. Figure 6 shows the FES applied to each muscle group during a typical trial of the far button pushing task and the resulting joint angle changes.

Figure 7 shows a patient's joint angles at each joint during unassisted and FES assisted trials at the far button pushing task. Improvements were seen in joint angles for all three joints with mean tracking accuracy improving by $54 \%, 59 \%$ and $66 \%$ for the shoulder, elbow and wrist, respectively. Furthermore, the patient was not able to successfully press the button during the unassisted trial, but was successful when the controlled FES was applied. The results demonstrate the success of the control system for improving movement accuracy during reaching and grasping tasks. Initial results from three participants that have completed 

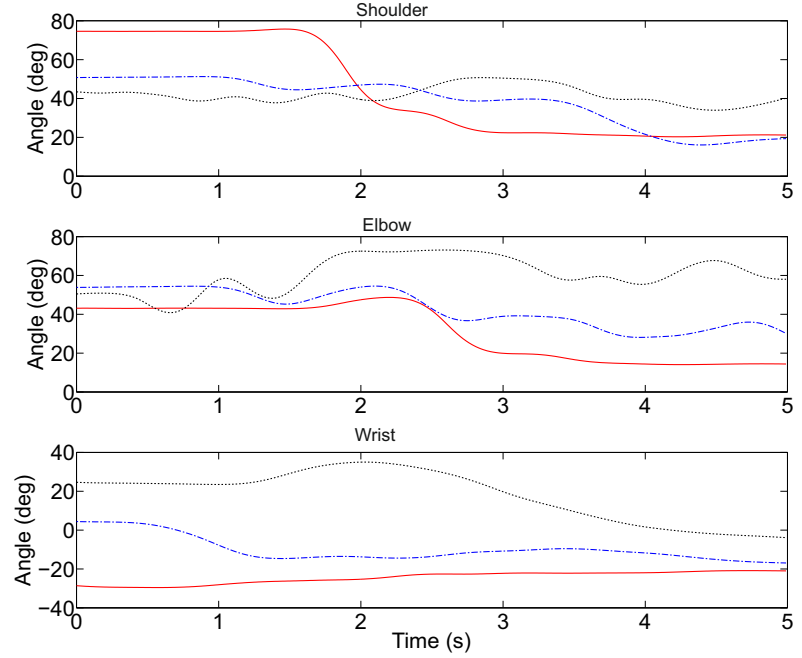

Fig. 7. An example of patient's performance for unassisted (fine dash, black), and FES assisted trials (large dash, blue) compared to the reference (solid, red) during the far button pushing task.

18 treatment sessions are encouraging, with all participants showing improvements in joint angle performance during unassisted tasks. Mean joint angle error across the three joints during unassisted performance reduced by between 35 and $51 \%$ over the sessions.

\section{CONCLUSION}

An upper-limb stroke rehabilitation system has been developed utilising ILC to regulate FES applied to extensors of the shoulder, elbow, wrist and hand joints during every-day functional tasks. The system employs the Kinect as a low cost non-contact method of collecting real-time kinematic data and provides assistance against gravity using a spring support. The system incorporates a range of functional tasks that challenge different aspects of reaching and grasping. The use of ILC to update the FES applied during trials encourages maximum effort from the patient during the movement, which along with the trial repetitions can assist with Hebbian learning. Initial results from current clinical trials indicate the success of the system for increasing patients' movement and controlling the FES during functional tasks incorporating real world reach and grasp activities. Future work on this project will focus on minimising the system and developing the software so that it may be used in patients' homes without the need for a therapist.

\section{ACKNOWLEDGEMENTS}

This work is supported by the Engineering and Physical Sciences Research Council Grant No. EP/I01909X/1, and Bridging The Gap.

\section{REFERENCES}

[1] D. of Health., Progress in Improving Stroke Care. National Audit Office, 2010

[2] H. T. Hendricks, J. van L.k, A. C. Geurts, and M. J. Zwarts, "Motor recovery after stroke: A systematic review of the literature," Archives of Physical Medicine and Rehabilitation, vol. 83, no. 11, pp. 1629 $1637,2002$.
[3] J. R. D. Kroon, J. H. van der Lee, M. J. Izerman, and G. J. Lankhorst, "Therapeutic electrical stimulation to improve motor control and functional abilities of the upper extremity after stroke: a systematic review," Clinical Rehabilitation, vol. 16, pp. 350-360, 2002.

[4] J. H. Burridge and M. Ladouceur, "Clinical and therapeutic applications of neuromuscular stimulation: A review of current use and speculation into future developments," Neuromodulation, vol. 4, no. 4, pp. 147-154, 2001.

[5] D. N. Rushton, "Functional electrical stimulation and rehabilitation an hypothesis," Medical Engineering and Physics, vol. 25, no. 1, pp. 75-78, 2003.

[6] C. T. Freeman, A. M. Hughes, J. H. Burridge, P. H. Chappell, P. L. Lewin, and E. Rogers, "Iterative learning control of FES applied to the upper extremity for rehabilitation," Control Engineering Practice, vol. 17 , no. 3, pp. 368-381, 2009.

[7] A. M. Hughes, C. T. Freeman, J. H. Burridge, P. H. Chappell, P. Lewin, and E. Rogers, "Feasibility of iterative learning control mediated by functional electrical stimulation for reaching after stroke," Journal of Neurorehabilitation and Neural Repair, vol. 23, no. 6, pp. 559-568, 2009.

[8] K. L. Meadmore, A. M. Hughes, C. T. Freeman, Z. Cai, D. Tong, J. H. Burridge, and E. Rogers, "Functional electrical stimulation mediated by iterative learning control and 3D robotics reduces motor impairment in chronic stroke," Journal of NeuroEngineering and Rehabilitation, vol. 9, no. 32, JUN 72012.

[9] Z. Cai, D. Tong, K. L. Meadmore, C. T. Freeman, A. M. Hughes, E. Rogers, and J. H. Burridge, "Design \& control of a $3 \mathrm{~d}$ stroke rehabilitation platform.” IEEE Int Conf Rehabil Robot, pp. 1-6, 2011.

[10] C. T. Freeman, E. Rogers, A.-M. Hughes, J. H. Burridge, and K. L. Meadmore, "Electrical Stimulation and Robotic-Assisted Upper Limb Stroke Rehabilitation," IEEE Control Systems Magazine, vol. 32, no. 1, pp. 18-43, 2012.

[11] C. T. Freeman, T. Exell, K. Meadmore, E. Hallewell, A.-M. Hughes, and J. Burridge, "Computational models of upper limb movement during functional reaching tasks for application in electrical stimulation based stroke rehabilitation," in 4th European Conference on Technically Assisted Rehabilitation, 2013.

[12] R. A. Clark, Y. H. Pua, K. Fortin, C. Ritchie, K. E. Webster, L. Denehy, and A. L. Bryant, "Validity of the microsoft kinect for assessment of postural control," Gait \& Posture, vol. 36, no. 3, pp. 372-377, 2012.

[13] T. Dutta, "Evaluation of the kinect sensor for 3-d kinematic measurement in the workplace," Applied Ergonomics, vol. 43, no. 4, pp. 645-649, 2012.

[14] J. R. D. Kroon, M. J. IJzerman, J. J. Chae, G. J. Lankhorst, and G. Zilvold, "Relation between stimulation characteristics and clinical outcome in studies using electrical stimulation to improve motor control of the upper extremity in stroke," Journal of Rehabilitation Medicine, vol. 37, no. 2, pp. 65-74, 2005.

[15] A. I. Soska, C. T. Freeman, and E. Rogers, "Optimal actuation site selection for surface electrode array based control of the wrist and hand," in IFAC International Workshop on Adaptation and Learning in Control and Signal Processing, 2013.

[16] M. Levin, "Interjoint coordination during pointing movements is disrupted in spastic hemiparesis," BRAIN, vol. 119, no. Part 1, pp. 281-293, FEB 1996.

[17] F. Le, I. Markovsky, C. T. Freeman, and E. Rogers, "Identification of electrically stimulated muscle models of stroke patients," Control Engineering Practice, vol. 18, pp. 396-407, 2010.

[18] C. T. Freeman, "Constrained point-to-point iterative learning control with experimental verification," Control Engineering Practice, vol. 20, no. 5, pp. 489-498, 2012.

[19] C. T. Freeman and Y. Tan, "Iterative learning control with mixed constraints for point-to-point tracking," IEEE Transactions on Control System Technology, vol. PP, no. 99, pp. 1-13, 2012.

[20] D. H. Owens, C. T. Freeman, and B. Chu, "Multivariable norm optimal iterative learning control with auxiliary optimization," International Journal of Control, vol. In Press, 2013.

[21] C. T. Freeman, A. M. Hughes, J. H. Burridge, P. H. Chappell, P. L. Lewin, and E. Rogers, "A model of the upper extremity using FES for stroke rehabilitation," Journal of Biomechanical Engineering, vol. 131, no. 3, pp. $031006-1-031006-10,2009$.

[22] C. T. Freeman, P. L. Lewin, and E. Rogers, "Experimental evaluation of iterative learning control algorithms for non-minimum phase plants," Int. Journal of Control, vol. 78, no. 11, pp. 826-846, 2005. 